2. Shaughnessy MK, Micielli RL, DePestel DD, et al. Evaluation of hospital room assignment and acquisition of Clostridium difficile infection. Infect Control Hosp Epidemiol 2011;32(3):201-206.

3. Best EL, Sandoe JAT, Wilcox MH. Potential for aerosolization of Clostridium difficile after flushing toilets: the role of toilet lids in reducing environmental contamination risk. J Hosp Infect 2012; $80(1): 1-5$.

\section{Survey on Influenza Vaccination Noncompliance among Nursing Students}

To the Editor-While annual influenza vaccination is strongly recommended for healthcare personnel (HCP), ${ }^{1}$ no specific evidence advocates nursing students to do the same. In a recent British study examining influenza vaccination among nursing students, the students lacked strong intention to receive vaccination. ${ }^{2}$ In Canada, many healthcare degree programs encourage seasonal influenza vaccination, but only $21 \%$ of programs require it. ${ }^{3}$ According to a 2008-2009 survey of deans representing 563 US schools of medicine and nursing regarding adherence to the Advisory Committee on Immunization Practices recommendations for HCP, only $19.2 \%$ of 439 schools of nursing required influenza vaccination, and nursing schools were less likely to provide students free influenza vaccination compared with medical schools $(31 \%$ vs $78 \%) .^{4}$ Given the lack of study on nursing student influenza vaccination, we examined our nursing students' noncompliance with HCP influenza vaccination recommendations.

Among 544 nursing students who participated the 20132014 University of Pittsburgh Medical Center (UPMC) influenza vaccination data collection survey (September 20, 2013-December 6, 2013), 161 students $(29.6 \%)$ who reported not obtaining the vaccination without a medical contradiction were selected as study subjects. To protect students' personal information, a group e-mail alias (2014flusurvey@list.pitt .edu) was created to contact subjects. To incentivize participation, a $\$ 5$ Starbucks gift card was offered to every thirtieth respondent. Using the Qualtrics survey system, the questionnaire was designed with 14 questions: 1 queried vaccination compliance, 1 elicited an alias name for anonymous incentive distribution, 4 collected demographic information, and 8 generated reasons for noncompliance. After approval by the University of Pittsburgh institutional review board, the survey link was sent using the group alias e-mail. The survey was conducted over 2 weeks (April 15-28, 2014), with 2 reminder e-mails.

Of the 161 students, 58 students (36\%) responded (i.e., 53 completed the survey, and 5 sent "I got the vaccination" email replies). Most respondents (79\%) reported receiving influenza vaccination; only 12 students $(21 \%)$ were noncompliant. These students were all female and aged 20-55 years (median, 21). All were engaged in a clinical practicum. Nine were undergraduates, 2 were MSN students, and 1 was a PhD student. Students chose many reasons as applicable for not getting the vaccine: healthy enough (ie, never get the flu; $58 \%$ ), possible side effects (eg, soreness; $25 \%$ ), inconvenience $(25 \%)$, sick after the previous vaccination $(17 \%)$, no time (17\%), and "don't like needles" (17\%). Among the students who reported not getting the vaccine, $67 \%$ acknowledged knowing a free influenza vaccination was available through the student health service.

Most noncompliant students (91\%) agreed that unvaccinated HCP (including nursing students) could be at risk of contracting influenza or transmitting the virus during patient care. Although $75 \%$ of noncompliant students disagreed that the influenza vaccine causes illness and $58 \%$ believed the influenza vaccination is effective in preventing influenza, 59\% disagreed nonetheless that nursing students should receive the annual vaccination, and $67 \%$ disagreed with mandatory influenza vaccination as a condition of employment. To promote improved compliance, these students suggested that more convenient times and locations should be made available (Table 1).

Although our survey generated only a $36 \%$ response rate, and only $21 \%$ of the respondents provided information about their noncompliance, we believe our study presented useful information about the annual influenza vaccination compliance among nursing students. In the 2013-2014 flu season, at least $78.9 \%$ of our students reported receiving the vaccination (418 students total; 372 from the UPMC influenza

тAвLE 1. Selected Responses to the Survey Question "I Would Get Vaccinated for Influenza This Coming Year If ..."

\title{
Response
}

There was a way to protect against all strains of the flu.

I was absolutely forced to .... Students living in dorms should get the vaccine because of crowded living conditions, but otherwise we should be allowed personal choice on the flu vaccine.

It was free and more convenient.

It was offered at a convenient time either in the nursing building or at clinical/the hospital.

I was told that ... you had to make an appointment at the student center, which was hard to do with a schedule that is Monday-Friday, with clinical and classes lasting until 7 p.m. at night most nights and starting at 5 a.m. I think the School of Nursing should have a fair that vaccinates all students, similar to how hospitals get all of their employees vaccinated.

Nоте. This question was adopted from the 2005 University of Pittsburgh Medical Center "How do you feel about the influenza vaccine?" survey. 
vaccination data collection survey and 46 from our survey), while the adherence of 103 nonresponsive students was unknown. Compared with the estimated $72 \%$ HCP influenza vaccination coverage in the United States during the 20122013 flu season, ${ }^{5}$ the $79 \%$ compliance rate among our nursing students is commensurate with and likely influenced by UPMC vaccination recommendations for students with clinical assignments.

The results of our survey may not be generalizable to students in other schools of nursing. We suspect that the low response rate $(36 \%)$ may reflect the overlap of the survey period with final exams and graduation. However, given that the average response rate to surveys of students' major concerns is $40 \%-50 \%$, a response rate around $30 \%$ may be average for a topic about which the students are less concerned. To avoid conducting a theme-overlapping survey, we targeted noncompliant students from the UPMC survey results. However, because many of these students actually did receive vaccinations (79\% of respondents), the UPMC survey structure (only 5 clinical assignment-related questions) might be inappropriate in selecting study subjects and perhaps contributed to the low response rate; vaccinated students likely ignored our survey requests. Additionally, we could not examine which factors induced more vaccinations among subject students during the gap period between the UPMC September-December 2013 survey and our April 2014 survey.

University policy on student vaccination governs every affiliated school; however, because of HCP-like clinical exposure, students in health science schools should be provided more support in obtaining influenza vaccination. As our students suggested, with their busy class and clinical practicum schedules, a more convenient time and location arrangement for free influenza vaccination would be helpful in improving student compliance. Concerning mandatory influenza vaccination for HCP as a condition of employment, ${ }^{6}$ our nursing students showed an unwelcome opinion. Education to encourage influenza vaccination should be provided to nursing students because of their involvement in patient care. In addition to ongoing emphasis on influenza vaccination, addressing students' concerns related to convenience would increase their willingness to obtain vaccination and their compliance.

\section{ACKNOWLEDGMENTS}

We thank Janeen LaForce (Department Coordinator, Health and Community Systems, School of Nursing, University of Pittsburgh) for arranging the group e-mail alias to protect student's personal information, Linda Holden (Associate Director for Student Services, School of Nursing, University of Pittsburgh) for sending a reminder e-mail, and the 58 responding students for their participation in our survey.

Financial support. A \$5 Starbucks gift-card for the thirtieth respondent was funded by JaHyun Kang's start-up research seed money provided by the School of Nursing, University of Pittsburgh.

Potential conflicts of interest. All authors report no conflicts of interest relevant to this article. All authors submitted the ICMJE Form for Disclosure of Potential Conflicts of Interest, and the conflicts that the editors consider relevant to this article are disclosed here.

\section{JaHyun Kang, PhD, MPH; ${ }^{1}$ Sandra J. Engberg, $\mathrm{PhD}{ }^{1}$ Carlene A. Muto, MD, $\mathrm{MS}^{2}$}

Affiliations: 1. School of Nursing, University of Pittsburgh, Pittsburgh, Pennsylvania; 2. Department of Infection Control and Hospital Epidemiology, University of Pittsburgh Medical Center Health System, Pittsburgh, Pennsylvania; and Division of Infectious Diseases, Department of Medicine, School of Medicine, University of Pittsburgh, Pittsburgh, Pennsylvania.

Address correspondence to JaHyun Kang, PhD, MPH, 415 Victoria Building, 3500 Victoria Street, Pittsburgh, PA 15261 (kangjh@pitt.edu).

Infect Control Hosp Epidemiol 2014;35(10):1316-1317

(C) 2014 by The Society for Healthcare Epidemiology of America. All rights reserved. 0899-823X/2014/3510-0022\$15.00. DOI: $10.1086 / 678076$

\section{REFERENCES}

1. Pearson ML, Bridges CB, Harper SA. Influenza vaccination of health-care personnel: recommendations of the Healthcare Infection Control Practices Advisory Committee (HICPAC) and the Advisory Committee on Immunization Practices (ACIP). MMWR Recomm Rep 2006;55(RR-2):1-16.

2. Cornally N, Ann Deasy E, McCarthey G, McAuley C, Moran J, Weathers E. Student nurses' intention to get the influenza vaccine. Br J Nurs 2013;22(21):1207-1211.

3. Pianosi K, Chobotuk T, Halperin BA, Halperin SA. Influenza immunization practices and policies for health care students in Canada. Can J Infect Dis Med Microbiol 2013;24(4):195-201.

4. Lindley MC, Lorick SA, Spinner JR, et al. Student vaccination requirements of U.S. health professional schools: a survey. Ann Intern Med 2011;154(6):391-400.

5. Centers for Disease Control and Prevention. Influenza vaccination coverage among health-care personnel-United States, 2012-13 influenza season. MMWR Morb Mortal Wkly Rep 2013; 62(38):781-786.

6. Rakita RM, Hagar BA, Crome P, Lammert JK. Mandatory influenza vaccination of healthcare workers: a 5-year study. Infect Control Hosp Epidemiol 2010;31(9):881-888.

\section{Cost Implications of Duplicative Influenza Polymerase Chain Reaction Testing During the 2013-2014 Influenza Season: The Price of Certainty}

To the Editor-During the recent influenza season (ie, October 2013-February 2014), 77 admitted adults with an influenza-like illness (ILI) were tested for influenza A or B by 1 or more methods in the emergency department. ${ }^{1}$ During this influenza season, influenza A (H1N1 and H3N2) were the predominant circulating strains in our area. Within influenza B strains, we do not differentiate the Yamagata versus the Victoria lineages. The initial point of care test in our emergency department is a rapid influenza diagnostic test 